\title{
Synchronisation of two different uncertain fractional-order chaotic systems with unknown parameters using a modified adaptive sliding-mode controller
}

\section{A. Othman Almatroud ${ }^{1 *}$}

${ }^{*}$ Correspondence:

othman_almatroud@yahoo.com ${ }^{1}$ Department of Mathematics,

Faculty of Science, University of Ha'il, Ha'il, Kingdom of Saudi Arabia

\begin{abstract}
This article investigates a modified adaptive sliding-mode controller to achieve synchronisation between two different fractional-order chaotic systems with fully unknown parameters. A suitable parameter updating law is designed to tackle the unknown parameters. For constructing the modified adaptive sliding-mode control, a simple sliding surface is designed and the stability of the suggested method is proved using Lyapunov stability theory. Finally, the proposed method is applied to gain chaos synchronisation between two different pairs of fractional-order chaotic systems with uncertain parameters. Numerical simulations are performed to demonstrate the robustness and efficiency of the proposed method.
\end{abstract}

PACS Codes: $05.45 .-a ; 47.52 .+j ;$ 89.75.- $-k$

Keywords: Sliding mode controller; Unknown parameter; Adaptive control; Fractional-order chaotic systems

\section{Introduction}

The essence of studying chaotic systems is to understand their structure and behaviour. These systems are deemed important as their study links between science and nature. One way to obtain a perspective glimpse of their complex dynamics is through their chaos control. However, chaos control is almost always an impossible task. This fact is due to the systems' unpredictable behaviour and sensitivity towards initial conditions. Hence, many arbitrary assumptions are necessary to achieve control in practical engineering problems, but this also implies a loss of important information. Systems characterised by a fractional order have attracted much attention in recent years. The ability of fractional-order modelling is to describe, mathematically and physically, phenomena of our real-world more rationally and carefully than the classical integer-order calculus [1-3], which has made them particularly well suited to research into nonlinear systems, particularly in physics and biology. Many phenomena show fractional-order dynamics, such as viscoelasticity and the polarisation of the dielectric as well as electrode-electrolyte [4, 5]. The synchroni-

(c) The Author(s) 2020. This article is licensed under a Creative Commons Attribution 4.0 International License, which permits use, sharing, adaptation, distribution and reproduction in any medium or format, as long as you give appropriate credit to the original author(s) and the source, provide a link to the Creative Commons licence, and indicate if changes were made. The images or other third party material in this article are included in the article's Creative Commons licence, unless indicated otherwise in a credit line to the material. If material is not included in the article's Creative Commons licence and your intended use is not permitted by statutory regulation or exceeds the permitted use, you will need to obtain permission directly from the copyright holder. To view a copy of this licence, visit http://creativecommons.org/licenses/by/4.0/. 
sation of fractional-order chaotic systems has been documented extensively and has been the centre of interest in recent years, given its potential applicability to diverse fields of physics and engineering sciences such as cryptography, information processing, chemical reactions and biological systems [6].

Various control techniques have been proposed for controlling and synchronising fractional-order chaotic systems; for instance in [7] the authors extended the applications of adaptive control to anti-synchronise different fractional-order chaotic and hyperchaotic dynamical systems. New results on adaptive synchronisation design for chaotic Arneodo system of incommensurate fractional order with unknown parameters based on the Lyapunov stability theory were introduced in [8]. The finite time robust synchronisation problem of a class of uncertain fractional chaotic/hyper-chaotic systems with a novel fractional sliding mode control technique was investigated in [9]. A novel fractional-order fuzzy sliding mode control strategy was developed to realise the deployment of the tethered satellite system (TSS) with input saturation in [10]. A novel robust predictive control strategy was proposed for the synchronisation of fractional-order time-delay chaotic systems in [11]. A new robust optimal control strategy was presented to synchronise a class of fractionalorder chaotic systems with unknown fractional orders and uncertain dynamics, and input nonlinearities were proposed in [12]. A new technique using a recurrent non-singleton type-2 sequential fuzzy neural network for synchronisation of the fractional-order chaotic systems with time-varying delay and uncertain dynamics was presented in [13]. The robust stochastic stabilisation problem for a class of fuzzy Markovian jump systems with time-varying delay and external disturbances via sliding mode control scheme was studied in [14]. The synchronisation issue for a family of time-delayed fractional-order complex dynamical networks (FCDNs) with time delay, unknown bounded uncertainty and disturbance was investigated in [15].

Fortunately, some existing methods for synchronising integer-order systems can be rigorously generalised to fractional-order systems. However, in practical engineering situations, system parameters are often unknown and variable. Therefore, there is much interest in effectively synchronising two fractional-order chaotic systems of undetermined parameters. This is very significant for both theoretical and practical research. Among the aforementioned methods, the adaptive sliding-mode control strategy is an efficient control method for synchronising fractional-order chaotic systems when some or all the system parameters are unknown. The fundamental features of this strategy include its fast response, robustness against perturbations, good transient performance and ease of implementation in real applications. The main contributions of this paper are: a technique for synchronising fractional-order chaotic systems which will serve the purpose of synchronisation of fractional-order as well as integer-order chaotic systems; a modified adaptive sliding-mode synchronisation approach that is relevant to fractional-order chaotic systems with unknown parameters; synchronisation controller and parameter-identification technique are designed based on the Lyapunov stability method to achieve the synchronisation of two different pairs of fractional-order chaotic systems using the proposed method. Computer simulations based on the Adams-Bashforth-Moulton method support the theoretical findings.

This paper is organised as follows: Sect. 2 describes the fractional calculus and its properties. Section 3 presents a step-by-step methodology for the proposed modified adaptive sliding-mode synchronisation controller design of fractional-order chaotic systems with 
unknown parameters. Two simulation examples are given to demonstrate the effectiveness of the proposed synchronisation schemes in Sects. 4, 5. Finally, a conclusion in Sect. 6 closes the work.

\section{Fractional calculus and its properties}

The concept of an integer-order integro-differential operator can be extended by the fractional-order integro-differential operator using a generalisable formulation, that is,

$$
{ }_{a} D_{t}^{p}= \begin{cases}\frac{d^{p}}{d t^{p}} & p>0 \\ 1 & p=0 \\ \int_{a}^{t}(d \tau)^{-p} & p<0\end{cases}
$$

where $p$ is the fractional order which could be a complex number, and $a, t$ symbolise the limits of the operation. There are many definitions of the fractional integral and derivative which have been used in the recent literature, precisely, the following three definitions: Grünwald-Letnikov, Riemann-Liouville and Caputo. The current study dealt with the Riemann-Liouville definition [16, 17], which was given by

$$
{ }_{a} D_{t}^{p} f(t)=\frac{d^{m}}{d t^{m}} J_{t}^{m-p} f(t), \quad p>0,
$$

where $m=\lceil p\rceil, J$ is the fractional Riemann-Liouville integral and

$$
J_{t}^{\varphi} \phi(t)=\frac{1}{\Gamma(\varphi)} \int_{0}^{t} \frac{\phi(v)}{(t-v)^{1-\varphi}} d v
$$

with $0<\varphi \leq 1$ and $\Gamma(\cdot)$ is the gamma function. For $r>n \geq 0, p$ and $q$ are integers such that $0 \leq p-1 \leq r<p$ and $0 \leq q-1 \leq n<q$. Then

$$
{ }_{a} D_{t}^{r}\left({ }_{a} D_{t}^{-m} f(t)\right)={ }_{a} D_{t}^{r-m} f(t) .
$$

For $r, m \geq 0$, there exist integers $p$ and $q$ such that $0 \leq p-1 \leq r<p$ and $0 \leq q-1 \leq m<q$. Then

$$
{ }_{a} D_{t}^{r}\left({ }_{a} D_{t}^{m} f(t)\right)={ }_{a} D_{t}^{r+m} f(t)-\sum_{j=1}^{m}\left[{ }_{a} D_{t}^{m-j} f(t)\right]_{t=a} \frac{(t-a)^{-r-j}}{\Gamma(1-r-j)} .
$$

\section{Modified adaptive sliding-mode synchronisation of fractional-order chaotic systems with unknown parameters}

Consider a drive system of the form

$$
D_{t}^{p} x=f(x)+F(x) \phi,
$$

where $x \in R^{n}$ is the state vector of the drive system, $f: R^{n} \rightarrow R^{n}$ is a continuous vector function, $F: R^{n} \rightarrow R^{n \times d}$ is a matrix function, and $\phi \in R^{d}$ is a parameter vector. The controlled response system is given by

$$
D_{t}^{p} y=g(y)+G(y) \theta+U,
$$


where $y \in R^{n}$ is the state vector of the response system, $g: R^{n} \rightarrow R^{n}$ is a continuous function, $G: R^{n} \rightarrow R^{n \times k}$ is a matrix function, $\theta \in R^{k}$ is a parameter vector and $U \in R^{n}$ is a controller. The dynamics of the synchronisation errors can be expressed as

$$
D_{t}^{p} e(t)=g(y)+G(y) \theta-f(x)-F(x) \phi+U,
$$

where $e=y-x$. Our aim is to design an adaptive sliding-mode controller $U$ that makes states of the response system follow those of the drive system asymptotically, so that

$$
\lim _{t \rightarrow \infty}\|e\|=\lim _{t \rightarrow \infty}\left\|y\left(t, y_{0}\right)-x\left(t, x_{0}\right)\right\|=0,
$$

where $\|\cdot\|$ denotes the Euclidean norm. In accordance with the design strategy for adaptive sliding-mode control, we choose an input signal vector as follows:

$$
\begin{aligned}
U= & f(x)+F(x) \phi-g(y)-G(y) \theta+D_{t}^{p-1}[F(x)(\hat{\phi}-\phi)-g(y)-G(y)(\hat{\theta}-\theta) \\
& \left.-\left(D_{t}^{p-1} e(t)\right) \frac{(t)^{-(p-1)-1}}{\Gamma(-(p-1))}-w(t) K\right]
\end{aligned}
$$

where $\hat{\phi}, \hat{\theta}$ are the estimated system parameters and $K>0$ is a positive gain vector. Substituting a particular expression for $U$ into the error dynamics (8) yields a form that is convenient for the forthcoming stability analysis:

$$
\begin{aligned}
D_{t}^{p} e(t)= & D_{t}^{p-1}\left[F(x)(\hat{\phi}-\phi)-G(y)(\hat{\theta}-\theta)-\left(D_{t}^{p-1} e(t)\right)\right. \\
& \left.\frac{(t)^{-(p-1)-1}}{\Gamma(-(p-1))}-w(t) K\right]
\end{aligned}
$$

where the control input is defined as

$$
w(t)= \begin{cases}w^{+}(t) & s(e) \geq 0, \\ w^{-}(t) & s(e)<0\end{cases}
$$

and $s=s(e)$ is a switching surface that forms the desirable sliding dynamics. The sliding surface can be defined as

$$
s(e)=C e,
$$

where $C$ is a positive constant. The following two conditions must be fulfilled on the sliding surface:

$$
s(e)=0 \quad \text { and } \quad \dot{s}(e)=0 .
$$

The second condition is indispensable to constrain the state trajectory to remain on the switching surface defined by $s(e)=0$. According to sliding-mode control theory, the sliding mode controller is designed as

$$
w(t)=\left[\frac{s}{|s|+\gamma}\right],
$$


where $\gamma$ is a positive real number. The resultant error dynamics then follows:

$$
\begin{aligned}
D_{t}^{p} e(t)= & D_{t}^{p-1}\left[F(x) \tilde{\phi}-G(y) \tilde{\theta}-\left(D_{t}^{p-1} e(t)\right)\right. \\
& \left.\frac{(t)^{-(p-1)-1}}{\Gamma(-(p-1))}-w(t) K\right] .
\end{aligned}
$$

The laws for updating parameters can be specified as follows:

$$
\begin{aligned}
& \dot{\tilde{\alpha}}=-[F(x)]^{T} \lambda, \\
& \dot{\tilde{\beta}}=\left[G(y)^{T}\right] \lambda,
\end{aligned}
$$

where $\lambda=s C^{T}$. The following theorem introduces the necessary conditions for verifying the stability of the error system in (16).

Theorem 1 Consider the error dynamics (16) for a system controlled by $U$ in (10) and following the parameter-update laws in (17). The error system is then stabilised at its equilibrium.

Proof Form a Lyapunov function as follows:

$$
V=\frac{1}{2}\left[s^{2}+\tilde{\phi}^{T} \tilde{\phi}+\tilde{\theta}^{T} \tilde{\theta}\right]
$$

where $\tilde{\phi}=\hat{\phi}-\phi$ and $\tilde{\theta}=\hat{\theta}-\theta$. The time derivative of (18) is

$$
\dot{V}=\left[s \dot{e}^{T} C^{T}+\tilde{\phi}^{T} \dot{\tilde{\phi}}+\tilde{\theta}^{T} \dot{\tilde{\theta}}\right] \text {. }
$$

Using (5) in (19), we obtain

$$
\dot{V}=s\left[D_{t}^{p-1}\left(D_{t}^{p} e\right)+\left(D_{t}^{p-1} e(t)\right) \frac{(t)^{-(p-1)-1}}{\Gamma(-(p-1))}\right]^{T} C^{T}+\tilde{\phi}^{T} \dot{\tilde{\phi}}+\tilde{\theta}^{T} \dot{\tilde{\theta}}
$$

From (16) and (19), we obtain

$$
\begin{aligned}
\dot{V}= & s\left[D _ { t } ^ { p - 1 } \left(D _ { t } ^ { p - 1 } \left[F(x) \tilde{\phi}-G(y) \tilde{\theta}-\left(D_{t}^{p-1} e(t)\right) \frac{(t)^{-(p-1)-1}}{\Gamma(-(p-1))}\right.\right.\right. \\
& \left.\left.\left.-\frac{s}{|s|+\gamma} K\right]\right)+\left(D_{t}^{p-1} e(t)\right) \frac{(t)^{-(p-1)-1}}{\Gamma(-(p-1))}\right]^{T} C^{T}+\tilde{\phi}^{T} \dot{\tilde{\phi}}+\tilde{\theta}^{T} \dot{\tilde{\theta}}
\end{aligned}
$$

since $\forall p \in[0,1],(1-p)>0$ and $(p-1)<0$. Now, using (4) and (17), (21) reduces to

$$
\begin{aligned}
\dot{V}= & s\left[\left(F(x) \tilde{\phi}-G(y) \tilde{\theta}-\left(D_{t}^{q-1} e(t)\right) \frac{(t)^{-(p-1)-1}}{\Gamma(-(p-1))}-\frac{s}{|s|+\gamma} K\right)\right. \\
& \left.+\left(D_{t}^{p-1} e(t)\right) \frac{(t)^{-(p-1)-1}}{\Gamma(-(p-1))}\right]^{T} C^{T}+\tilde{\phi}^{T} \dot{\tilde{\phi}}+\tilde{\theta}^{T} \dot{\tilde{\theta}}, \\
\dot{V}= & s\left(F(x) \tilde{\phi}-G(y) \tilde{\theta}-\frac{s}{|s|+\gamma} K\right)^{T} C^{T}-\tilde{\phi}^{T} F(x)^{T} \lambda+\tilde{\theta}^{T} G(y)^{T} \lambda .
\end{aligned}
$$


Then (23) yields

$$
\dot{V}=-C K\left[\frac{s^{2}}{|s|+\gamma}\right]<0 .
$$

Since both $s^{2}>0$ and $|s|>0$, then, when $e \neq 0$ and $C K>0$, the inequality $\dot{V}<0$ holds. Hence, $V$ is positive-definite and $\dot{V}$ is negative-definite. Then the error system is stable in the sense defined in Lyapunov stability theory [18] and the response system (7) is synchronised with the drive system (6) globally and asymptotically. This completes the proof.

\section{Synchronisation between fractional-order Genesio-Tesi and Lü chaotic systems using the modified adaptive sliding-mode control}

In this section, we investigate the synchronisation behaviour between the fractional-order Genesio-Tesi [19, 20] and Lü [21] systems using the modified adaptive sliding-mode control method. The fractional-order Genesio-Tesi system is assumed to drive the fractionalorder Lü system. Both systems are defined with unknown parameters:

$$
\begin{aligned}
& D_{t}^{p_{1}} x_{1}=y_{1}, \\
& D_{t}^{p_{2}} y_{1}=z_{1}, \\
& D_{t}^{p_{3}} z_{1}=-a_{1} x_{1}-b_{1} y_{1}-c_{1} z_{1}+d_{1} x_{1}^{2},
\end{aligned}
$$

and

$$
\begin{aligned}
& D_{t}^{p_{1}} x_{2}=a_{2}\left(y_{2}-x_{2}\right)+u_{1}, \\
& D_{t}^{p_{2}} y_{2}=-x_{2} z_{2}+b_{2} y_{2}+u_{2}, \\
& D_{t}^{p_{3}} z_{2}=x_{2} y_{2}-c_{2} z_{2}+u_{3},
\end{aligned}
$$

where $\left(u_{1}, u_{2}, u_{3}\right)^{T}$ is the controller function to be designed. Let the vector error state be $e=$ $y-x$. Then the error dynamical system between the drive (25) and response (26) systems can be written as

$$
\begin{aligned}
& D_{t}^{p_{1}} e_{1}(t)=a_{2}\left(y_{2}-x_{2}\right)-y_{1}+u_{1}, \\
& D_{t}^{p_{2}} e_{2}(t)=-x_{2} z_{2}+b_{2} y_{2}-z_{1}+u_{2}, \\
& D_{t}^{p_{3}} e_{3}(t)=x_{2} y_{2}-c_{2} z_{2}+a_{1} x_{1}+b_{1} y_{1}+c_{1} z_{1}-d_{1} x_{1}^{2}+u_{3} .
\end{aligned}
$$

The aim of the modified adaptive sliding-mode control method is to formulate a controller function $U$ capable of synchronising the states of the response and drive systems with fully unknown parameters. Then, the switching surface is described as follows:

$$
\begin{aligned}
& s(e)=e_{1}+e_{2}+e_{3}, \\
& w(t)=\frac{s}{|s|+0.01},
\end{aligned}
$$


where the control parameters are set to $C=(1,1,1), K=(3,0,11)^{T}$ and $\gamma=0.01$. The adaptive sliding-mode controller of (27) can be determined as follows:

$$
\begin{aligned}
u_{1}= & -a_{2}\left(y_{2}-x_{2}\right)+y_{1}+D_{t}^{p_{1}-1}\left[-\hat{a}_{2}\left(y_{2}-x_{2}\right)-\left(D_{t}^{p_{1}-1} e_{1}(t)\right)\right. \\
& \left.\frac{(t)^{-\left(p_{1}-1\right)-1}}{\Gamma\left(-\left(p_{1}-1\right)\right)}-\frac{3 s}{|s|+0.01}\right], \\
u_{2}= & x_{2} z_{2}-b_{2} y_{2}+z_{1}+D_{t}^{p_{2}-1}\left[-\hat{b}_{2} y_{2}-\left(D_{t}^{p_{2}-1} e_{2}(t)\right) \frac{(t)^{-\left(p_{2}-1\right)-1}}{\Gamma\left(-\left(p_{2}-1\right)\right)}\right], \\
u_{3}= & -x_{2} y_{2}+c_{2} z_{2}-a_{1} x_{1}-b_{1} y_{1}-c_{1} z_{1}+d_{1} x_{1}^{2}+D_{t}^{p_{3}-1}\left[\hat{c}_{2} z_{2}-\hat{a}_{1} x_{1}\right. \\
& \left.\quad \hat{b}_{1} y_{1}-\hat{c}_{1} z_{1}+\hat{d}_{1} x_{1}^{2}-\left(D_{t}^{p_{3}-1} e_{3}(t)\right) \frac{(t)^{-\left(p_{3}-1\right)-1}}{\Gamma\left(-\left(p_{3}-1\right)\right)}-\frac{11 s}{|s|+0.01}\right],
\end{aligned}
$$

and the estimates $\hat{a}_{1}, \hat{b}_{1}, \hat{c}_{1}, \hat{d}_{1}, \hat{a}_{2}, \hat{b}_{2}$ and $\hat{c}_{2}$ are updated, respectively, according to the following algorithm:

$$
\begin{aligned}
& \dot{\tilde{a}}_{1}=x_{1} s, \\
& \dot{\tilde{b}}_{1}=y_{1} s, \\
& \dot{\tilde{c}}_{1}=z_{1} s, \\
& \dot{\tilde{d}}_{1}=-x_{s}^{2} s, \\
& \dot{\tilde{a}}_{2}=\left(y_{2}-x_{2}\right) s, \\
& \dot{\tilde{b}}_{2}=y_{2} s, \\
& \dot{\tilde{c}}_{2}=-z_{2} s .
\end{aligned}
$$

Theorem 2 Consider the error dynamics (27). If this system is controlled by $u_{i}=\left(u_{1}, u_{2}\right.$, $\left.u_{3}\right)^{T}, i=1,2,3$, in (29) with the adaptive laws in (30), then the error system is stabilised at its equilibrium.

Proof Inserting (29) in (27) yields the following error system:

$$
\begin{aligned}
D_{t}^{p_{1}} e_{1}(t)= & D_{t}^{p_{1}-1}\left[-\tilde{a}_{2}\left(y_{2}-x_{2}\right)-\left(D_{t}^{p_{1}-1} e_{1}(t)\right) \frac{(t)^{-\left(p_{1}-1\right)-1}}{\Gamma\left(-\left(p_{1}-1\right)\right)}\right. \\
& \left.-\frac{3 s}{|s|+0.01}\right], \\
D_{t}^{p_{2}} e_{2}(t)= & D_{t}^{p_{2}-1}\left[-\tilde{b}_{2} y_{2}-\left(D_{t}^{p_{2}-1} e_{2}(t)\right) \frac{(t)^{-\left(p_{2}-1\right)-1}}{\Gamma\left(-\left(p_{2}-1\right)\right)}\right] \\
D_{t}^{p_{3}} e_{3}(t)= & D_{t}^{p_{3}-1}\left[\tilde{c}_{2} z_{2}-\tilde{a}_{1} x_{1}-\tilde{b}_{1} y_{1}-\tilde{c}_{1} z_{1}+\tilde{d}_{1} x_{1}^{2}-\left(D_{t}^{p_{3}-1} e_{3}(t)\right)\right. \\
& \left.\frac{(t)^{-\left(p_{3}-1\right)-1}}{\Gamma\left(-\left(p_{3}-1\right)\right)}-\frac{11 s}{|s|+0.01}\right]
\end{aligned}
$$


where $\tilde{a}_{1}=\hat{a}_{1}-a_{1}, \tilde{b}_{1}=\hat{b}_{1}-b_{1}, \tilde{c}_{1}=\hat{c}_{1}-c_{1}, \tilde{d}_{1}=\hat{d}_{1}-d_{1}, \tilde{a}_{2}=\hat{a}_{2}-a_{2}, \tilde{b}_{2}=\hat{b}_{2}-b_{2}$ and $\tilde{c}_{2}=\hat{c}_{2}-c_{2}$. Consider the following Lyapunov function candidate:

$$
V=\frac{1}{2}\left(s^{2}+\tilde{a}_{1}^{2}+\tilde{b}_{1}^{2}+\tilde{c}_{1}^{2}+\tilde{d}_{1}^{2}+\tilde{a}_{2}^{2}+\tilde{b}_{2}^{2}+\tilde{c}_{2}^{2}\right)
$$

Differentiating (32) with respect to time using (30) and (5) yields

$$
\begin{aligned}
& \dot{V}=\left(s \dot{s}+\tilde{a}_{1} \dot{\tilde{a}}_{1}+\tilde{b}_{1} \dot{\tilde{b}}_{1}+\tilde{c}_{1} \dot{\tilde{c}}_{1}+\tilde{d}_{1} \dot{\tilde{d}}_{1}+\tilde{a}_{2} \dot{\tilde{a}}_{2}+\tilde{b}_{2} \dot{\tilde{b}}_{2}+\tilde{c}_{2} \dot{\tilde{c}}_{2}\right) \\
& =\left(s\left[D_{t}^{1-p_{1}}\left(D_{t}^{p_{1}} e_{1}(t)\right)+\left(D_{t}^{p_{1}-1} e_{1}(t)\right) \frac{(t)^{-\left(p_{1}-1\right)-1}}{\Gamma\left(-\left(p_{1}-1\right)\right)}\right]+s\left[D_{t}^{1-p_{2}}\left(D_{t}^{p_{2}} e_{2}(t)\right)\right.\right. \\
& \left.+\left(D_{t}^{p_{2}-1} e_{2}(t)\right) \frac{(t)^{-\left(p_{2}-1\right)-1}}{\Gamma\left(-\left(p_{2}-1\right)\right)}\right]+s\left[D_{t}^{1-p_{3}}\left(D_{t}^{p_{3}} e_{3}(t)\right)+\left(D_{t}^{p_{3}-1} e_{3}(t)\right)\right. \\
& \left.\left.\frac{(t)^{-\left(p_{3}-1\right)-1}}{\Gamma\left(-\left(p_{3}-1\right)\right)}\right]+\tilde{a}_{1} \dot{\tilde{a}}_{1}+\tilde{b}_{1} \dot{\tilde{b}}_{1}+\tilde{c}_{1} \dot{\tilde{c}}_{1}+\tilde{c}_{2} \dot{\tilde{c}}_{2}+\tilde{d}_{1} \dot{\tilde{d}}_{1}+\tilde{a}_{2} \dot{\tilde{a}}_{2}+\tilde{b}_{2} \dot{\tilde{b}}_{2}\right) \\
& =s\left[D_{t}^{1-p_{1}}\left(D_{t}^{p_{1}-1}\left[-\tilde{a}_{2}\left(y_{2}-x_{2}\right)-\left(D_{t}^{p_{1}-1} e_{1}(t)\right) \frac{(t)^{-\left(p_{1}-1\right)-1}}{\Gamma\left(-\left(p_{1}-1\right)\right)}-\frac{3 s}{|s|+0.01}\right]\right)\right. \\
& \left.+\left(D_{t}^{p_{1}-1} e_{1}(t)\right) \frac{(t)^{-\left(p_{1}-1\right)-1}}{\Gamma\left(-\left(p_{1}-1\right)\right)}\right]+s\left[D _ { t } ^ { 1 - p _ { 2 } } \left(D _ { t } ^ { p _ { 2 } - 1 } \left[-\tilde{b}_{2} y_{2}-\left(D_{t}^{p_{2}-1} e_{2}(t)\right)\right.\right.\right. \\
& \left.\left.\left.\frac{(t)^{-\left(p_{2}-1\right)-1}}{\Gamma\left(-\left(p_{2}-1\right)\right)}\right]\right)+\left(D_{t}^{p_{2}-1} e_{2}(t)\right) \frac{(t)^{-\left(p_{2}-1\right)-1}}{\Gamma\left(-\left(p_{2}-1\right)\right)}\right]+s\left[D _ { t } ^ { 1 - p _ { 3 } } \left(D _ { t } ^ { 1 - p _ { 3 } } \left[\tilde{c}_{2} z_{2}\right.\right.\right. \\
& \left.\left.-\tilde{a}_{1} x_{1}-\tilde{b}_{1} y_{1}-\tilde{c}_{1} z_{1}+\tilde{d}_{1} x_{1}^{2}-\left(D_{t}^{p_{3}-1} e_{3}(t)\right) \frac{(t)^{-\left(p_{3}-1\right)-1}}{\Gamma\left(-\left(p_{3}-1\right)\right)}-\frac{11 s}{|s|+0.01}\right]\right) \\
& \left.+\left(D_{t}^{p_{3}-1} e_{3}(t)\right) \frac{(t)^{-\left(p_{3}-1\right)-1}}{\Gamma\left(-\left(p_{3}-1\right)\right)}\right]+\tilde{a}_{1} \dot{\tilde{a}}_{1}+\tilde{b}_{1} \dot{\tilde{b}}_{1} \tilde{c}_{1} \dot{\tilde{c}}_{1}+\tilde{d}_{1} \dot{\tilde{d}}_{1}+\tilde{a}_{2} \dot{\tilde{a}}_{2}+\tilde{b}_{2} \dot{\tilde{b}}_{2}+\tilde{c}_{2} \dot{\tilde{c}}_{2}
\end{aligned}
$$

since $\forall p \in[0,1],(1-p)>0$ and $(p-1)<0$. Now, using (4) and (30), (33) reduces to

$$
\begin{aligned}
\dot{V}= & s\left(-\tilde{a}_{2}\left(y_{2}-x_{2}\right)-\frac{3 s}{|s|+0.01}\right)+s\left(-\tilde{b}_{2} y_{2}\right)+s\left(\tilde{c}_{2} z_{2}-\tilde{a}_{1} x_{1}\right. \\
& \left.-\tilde{b}_{1} y_{1}-\tilde{c}_{1} z_{1}+\tilde{d}_{1} x_{1}^{2}-\frac{11 s}{|s|+0.01}\right)+\tilde{a}_{1}\left(x_{1} s\right)+\tilde{b}_{1}\left(y_{1} s\right)+\tilde{c}_{1}\left(z_{1} s\right) \\
& +\tilde{d}_{1}\left(-x_{s}^{2} s\right)+\tilde{a}_{2}\left(\left(y_{2}-x_{2}\right) s\right)+\tilde{b}_{2}\left(y_{2} s\right)+\tilde{c}_{2}\left(-z_{2} s\right) .
\end{aligned}
$$

Then (34) reduces to

$$
\dot{V}=-\frac{14 s^{2}}{|s|+0.01} .
$$

Since both $s^{2}>0$ and $|s|>0$, then, when $e \neq 0, \dot{V}<0$ and hence $V$ is positive-definite while $\dot{V}$ is negative-definite, so the error system is stable according to the Lyapunov stability theory [18], and the response system (26) is synchronised with the drive system (25) globally and asymptotically. This completes the proof.

For simulations, we use the Adams-Bashforth-Moulton method to solve systems for the fractional order $p_{i}=0.95, i=1,2,3$. The uncertain parameters are set to $a_{1}=6, b_{1}=2.92$, 
(a)
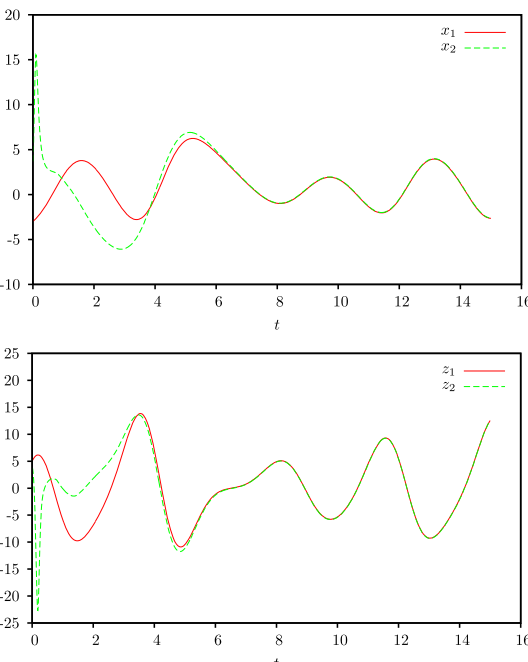

(c)

Figure 1 State trajectories of drive system (25) and response system (25): (a) signals $x_{1}$ and $x_{2}$; (b) signals $y_{1}$ and $y_{2} ;$ (c) signals $z_{1}$ and $z_{2} ;(\mathbf{d})$ evolution of the errors $e_{1}, e_{2}, e_{3}$ with respect to time $t$

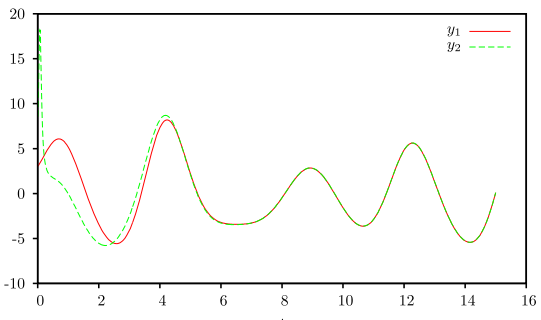

(b)

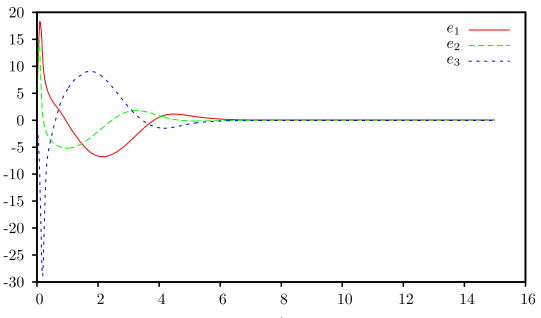

(d)

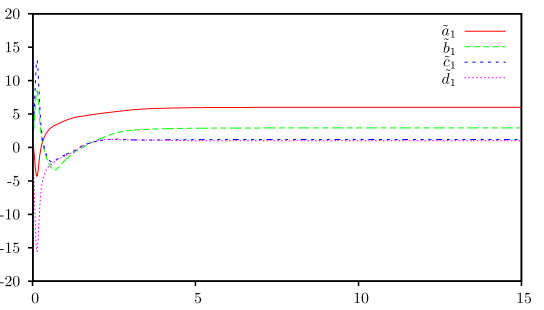

(a)

Figure 2 State trajectories of the adaptive parameters: (a) $\tilde{a}_{1}, \tilde{b}_{1}, \tilde{c}_{1}, \tilde{d}_{1} ;(\mathbf{b}) \tilde{a}_{2}, \tilde{b}_{2}, \tilde{c}_{2}$

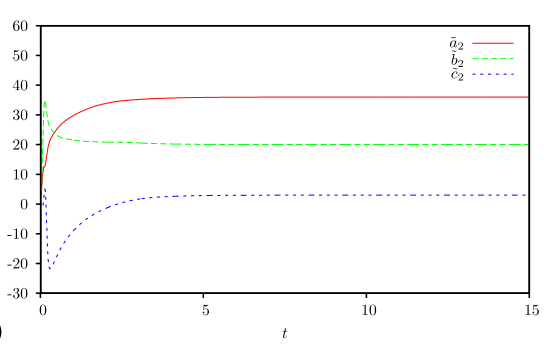

(b)

$c_{1}=1.2$ and $d_{1}:=1$; and $a_{2}=36, b_{2}=20, c_{2}=3$. The initial values of the fractional-order drive and response systems (25)-(26) and the estimated parameters are respectively and arbitrarily set in simulations to $x_{1}(0)=-3, \mathrm{y}_{1}(0)=3, z_{1}(0)=5, x_{2}(0)=2, y_{2}(0)=7$ and $z_{2}(0)=4$; and $\tilde{a}_{1}(0)=1, \tilde{b}_{1}(0)=1, \tilde{c}_{1}(0)=1, \tilde{d}_{1}(0)=1, \tilde{a}_{2}(0)=1$ and $\tilde{b}_{2}(0)=1$. Figures $1-2$ depict the modified adaptive sliding-mode synchronisation of systems (25)-(26) via the adaptive control laws (29) and (30). Figure 1(a)-(c) displays the steady-state plane trajectories of the drive (25) and response (26) systems. Figure 1(d) displays the synchronisation errors $e_{1}, e_{2}$ and $e_{3}$ as functions of time $t$. Figure 2(a)-(b) displays the temporal response of the estimated parameter values $\tilde{a}_{1}, \tilde{b}_{1}, \tilde{c}_{1}, \tilde{d}_{1}, \tilde{a}_{2}$ and $\tilde{b}_{2}$ of the drive (25) and response (26) systems.

\section{Synchronisation of fractional-order Chen and Lü chaotic systems using the modified adaptive sliding-mode control method}

This section considers the synchronisation behaviour between the fractional-order Chen [22] and Lü systems using the modified adaptive sliding-mode control method. Now let us assume that the fractional-order Chen system is a drive system and the fractional-order Lü system is considered as a response system. In terms of unknown parameters, the drive 
system is specified as

$$
\begin{aligned}
& D_{t}^{p_{1}} x_{1}=a_{1}\left(y_{1}-x_{1}\right), \\
& D_{t}^{p_{2}} y_{1}=\left(b_{1}-a_{1}\right) x_{1}-x_{1} z_{1}+b_{1} y_{1}, \\
& D_{t}^{p_{3}} z_{1}=x_{1} y_{1}-c_{1} z_{1},
\end{aligned}
$$

and the response system as

$$
\begin{aligned}
& D_{t}^{p_{1}} x_{2}=a_{2}\left(y_{2}-x_{2}\right)+u_{1}, \\
& D_{t}^{p_{2}} y_{2}=-x_{2} z_{2}+b_{2} y_{2}+u_{2}, \\
& D_{t}^{p_{3}} z_{2}=x_{2} y_{2}-c_{2} z_{2}+u_{3},
\end{aligned}
$$

where $\left(u_{1}, u_{2}, u_{3}\right)^{T}$ is the controller to be specified. Let the vector error state be $e=y-x$. Hence, the error dynamical system between the response system (26) and the drive system (25) is given by

$$
\begin{aligned}
& D_{t}^{p_{1}} e_{1}(t)=a_{2}\left(y_{2}-x_{2}\right)-a_{1}\left(y_{1}-x_{1}\right)+u_{1}, \\
& D_{t}^{p_{2}} e_{2}(t)=-x_{2} z_{2}+b_{2} y_{2}-\left(b_{1}-a_{1}\right) x_{1}+x_{1} z_{1}-b_{1} y_{1}+u_{2}, \\
& D_{t}^{p_{3}} e_{3}(t)=x_{2} y_{2}-c_{2} z_{2}-x_{1} y_{1}+c_{1} z_{1}+u_{3} .
\end{aligned}
$$

The aim of the modified adaptive sliding-mode control method is to design a controller $U$ such that the states of the response and the drive systems are synchronised with fully unknown parameters. Then the switching surface is described as

$$
\begin{aligned}
& s(e)=e_{1}+e_{2}+e_{3}, \\
& w(t)=\frac{s}{|s|+0.01},
\end{aligned}
$$

with the control parameters set to $C=(1,1,1), K=(0,0,15)^{T}$ and $\gamma=0.01$. The adaptive sliding-mode controller of (38) can be determined as follows:

$$
\begin{aligned}
u_{1}= & a_{1}\left(y_{1}-x_{1}\right)-a_{2}\left(y_{2}-x_{2}\right)+D_{t}^{p_{1}-1}\left[\hat{a}_{1}\left(y_{1}-x_{1}\right)-\hat{a}_{2}\left(y_{2}-x_{2}\right)\right) \\
& \left.-\left(D_{t}^{p_{1}-1} e_{1}(t)\right) \frac{(t)^{-\left(p_{1}-1\right)-1}}{\Gamma\left(-\left(p_{1}-1\right)\right)}\right], \\
u_{2}= & x_{2} z_{2}-b_{2} y_{2}+\left(b_{1}-a_{1}\right) x_{1}-x_{1} z_{1}+b_{1} y_{1}+D_{t}^{p_{2}-1}\left[-\hat{b}_{2} y_{2}+\left(\hat{b}_{1}-\hat{a}_{1}\right) x_{1}\right. \\
& \left.+\hat{b}_{1} y_{1}-\left(D_{t}^{p_{2}-1} e_{2}(t)\right) \frac{(t)^{-\left(p_{2}-1\right)-1}}{\Gamma\left(-\left(p_{2}-1\right)\right)}\right], \\
u_{3}= & -x_{2} y_{2}+c_{2} z_{2}+x_{1} y_{1}-c_{1} z_{1}+D_{t}^{p_{3}-1}\left[\hat{c}_{2} z_{2}-\hat{c}_{1} z_{1}-\left(D_{t}^{p_{3}-1} e_{3}(t)\right) \frac{(t)^{-\left(p_{3}-1\right)-1}}{\Gamma\left(-\left(p_{3}-1\right)\right)}\right. \\
& \left.-\frac{15 s}{|s|+0.01}\right]
\end{aligned}
$$


and the estimates $\hat{a}_{1}, \hat{b}_{1}, \hat{c}_{1}, \hat{a}_{2}, \hat{b}_{2}$ and $\hat{c}_{2}$ are updated according to the following algorithm:

$$
\begin{aligned}
& \dot{\tilde{a}}_{1}=-\left(y_{1}-x_{1}\right) s+x_{1} s, \\
& \dot{\tilde{b}}_{1}=-\left(x_{1}+y_{1}\right) s, \\
& \dot{\tilde{c}}_{1}=z_{1} s, \\
& \dot{\tilde{a}}_{2}=\left(y_{2}-x_{2}\right) s, \\
& \dot{\tilde{b}}_{2}=y_{2} s, \\
& \dot{\tilde{c}}_{2}=-z_{2} s .
\end{aligned}
$$

Theorem 3 Consider the error dynamics (38). If this system is controlled by $u_{i}=\left(u_{1}, u_{2}\right.$, $\left.u_{3}\right)^{T}, i=1,2,3$, in (40) with the adaptive laws in (41), then the error system is stabilised at its equilibrium.

Proof Inserting (40) in (38) yields the following error system:

$$
\begin{aligned}
D_{t}^{p_{1}} e_{1}(t)= & D_{t}^{p_{1}-1}\left[\tilde{a}_{1}\left(y_{1}-x_{1}\right)-\tilde{a}_{2}\left(y_{2}-x_{2}\right)-\left(D_{t}^{p_{1}-1} e_{1}(t)\right)\right. \\
& \left.\frac{\left.(t)^{-\left(p_{1}-1\right)-1}\right]}{\Gamma\left(-\left(p_{1}-1\right)\right)}\right] \\
D_{t}^{p_{2}} e_{2}(t)= & D_{t}^{p_{2}-1}\left[-\tilde{b}_{2} y_{2}+\left(\tilde{b}_{1}-\tilde{a}_{1}\right) x_{1}+\tilde{b}_{1} y_{1}-\left(D_{t}^{p_{2}-1} e_{2}(t)\right) \frac{(t)^{-\left(p_{2}-1\right)-1}}{\Gamma\left(-\left(p_{2}-1\right)\right)}\right] \\
D_{t}^{p_{3}} e_{3}(t)= & D_{t}^{p_{3}-1}\left[\tilde{c}_{2} z_{2}-\tilde{c}_{1} z_{1}-\left(D_{t}^{p_{3}-1} e_{3}(t)\right)\right. \\
& \left.\frac{(t)^{-\left(p_{3}-1\right)-1}}{\Gamma\left(-\left(p_{3}-1\right)\right)}-\frac{15 s}{|s|+0.01}\right]
\end{aligned}
$$

where $\tilde{a}_{1}=\hat{a}_{1}-a_{1}, \tilde{b}_{1}=\hat{b}_{1}-b_{1}, \tilde{c}_{1}=\hat{c}_{1}-c_{1}, \tilde{a}_{2}=\hat{a}_{2}-a_{2}, \tilde{b}_{2}=\hat{b}_{2}-b_{2}$ and $\tilde{c}_{2}=\hat{c}_{2}-c_{2}$. Considering the Lyapunov function candidate

$$
V=\frac{1}{2}\left(s^{2} s+\tilde{a}_{1}^{2}+\tilde{b}_{1}^{2}+\tilde{c}_{1}^{2}+\tilde{a}_{2}^{2}+\tilde{b}_{2}^{2}+\tilde{c}_{2}^{2}\right)
$$

differentiating (43) with respect to time using (41) and (5) yields

$$
\begin{aligned}
\dot{V}= & \left(s \dot{s}+\tilde{a}_{1} \dot{\tilde{a}}_{1}+\tilde{b}_{1} \dot{\tilde{b}}_{1}+\tilde{c}_{1} \dot{\tilde{c}}_{1}+\tilde{a}_{2} \dot{\tilde{a}}_{2}+\tilde{b}_{2} \dot{\tilde{b}}_{2}+\tilde{c}_{2} \dot{\tilde{c}}_{2}\right) \\
= & \left(s\left[D_{t}^{1-p_{1}}\left(D_{t}^{p_{1}} e_{1}(t)\right)+\left(D_{t}^{p_{1}-1} e_{1}(t)\right) \frac{(t)^{-\left(p_{1}-1\right)-1}}{\Gamma\left(-\left(p_{1}-1\right)\right)}\right]+s\left[D_{t}^{1-p_{2}}\left(D_{t}^{p_{2}} e_{2}(t)\right)\right.\right. \\
& \left.+\left(D_{t}^{p_{2}-1} e_{2}(t)\right) \frac{(t)^{-\left(p_{2}-1\right)-1}}{\Gamma\left(-\left(p_{2}-1\right)\right)}\right]+s\left[D_{t}^{1-p_{3}}\left(D_{t}^{p_{3}} e_{3}(t)\right)+\left(D_{t}^{p_{3}-1} e_{3}(t)\right)\right. \\
& \left.\left.\frac{(t)^{-\left(p_{3}-1\right)-1}}{\Gamma\left(-\left(p_{3}-1\right)\right)}\right]+\tilde{a}_{1} \dot{\tilde{a}}_{1}+\tilde{b}_{1} \dot{\tilde{b}}_{1}+\tilde{c}_{1} \dot{\tilde{c}}_{1}+\tilde{c}_{2} \dot{\tilde{c}}_{2}+\tilde{a}_{2} \dot{\tilde{a}}_{2}+\tilde{b}_{2} \dot{\tilde{b}}_{2}\right) \\
= & s\left[D_{t}^{1-p_{1}}\left(D_{t}^{p_{1}-1}\left[\tilde{a}_{1}\left(y_{1}-x_{1}\right)-\tilde{a}_{2}\left(y_{2}-x_{2}\right)-\left(D_{t}^{p_{1}-1} e_{1}(t)\right) \frac{(t)^{-\left(p_{1}-1\right)-1}}{\Gamma\left(-\left(p_{1}-1\right)\right)}\right]\right)\right.
\end{aligned}
$$




$$
\begin{aligned}
& \left.+\left(D_{t}^{p_{1}-1} e_{1}(t)\right) \frac{(t)^{-\left(p_{1}-1\right)-1}}{\Gamma\left(-\left(p_{1}-1\right)\right)}\right]+s\left[D _ { t } ^ { 1 - p _ { 2 } } \left(D _ { t } ^ { p _ { 2 } - 1 } \left[-\tilde{b}_{2} y_{2}+\left(\tilde{b}_{1}-\tilde{a}_{1}\right) x_{1}+\tilde{b}_{1} y_{1}\right.\right.\right. \\
& \left.\left.\left.-\left(D_{t}^{p_{2}-1} e_{2}(t)\right) \frac{(t)^{-\left(p_{2}-1\right)-1}}{\Gamma\left(-\left(p_{2}-1\right)\right)}\right]\right)+\left(D_{t}^{p_{2}-1} e_{2}(t)\right) \frac{(t)^{-\left(p_{2}-1\right)-1}}{\Gamma\left(-\left(p_{2}-1\right)\right)}\right] \\
& +s\left[D_{t}^{1-p_{3}}\left(D_{t}^{1-p_{3}}\left[\tilde{c}_{2} z_{2}-\tilde{c}_{1} z_{1}-\left(D_{t}^{p_{3}-1} e_{3}(t)\right) \frac{(t)^{-\left(p_{3}-1\right)-1}}{\Gamma\left(-\left(p_{3}-1\right)\right)}-\frac{15 s}{|s|+0.01}\right]\right)\right. \\
& \left.+\left(D_{t}^{p_{3}-1} e_{3}(t)\right) \frac{(t)^{-\left(p_{3}-1\right)-1}}{\Gamma\left(-\left(p_{3}-1\right)\right)}\right]+\tilde{a}_{1} \dot{\tilde{a}}_{1}+\tilde{b}_{1} \dot{\tilde{b}}_{1}+\tilde{c}_{1} \dot{\tilde{c}}_{1}+\tilde{a}_{2} \dot{\tilde{a}}_{2}+\tilde{b}_{2} \dot{\tilde{b}}_{2}+\tilde{c}_{2} \dot{\tilde{c}}_{2}
\end{aligned}
$$

since $\forall p \in[0,1],(1-p)>0$ and $(p-1)<0$. Now, using (4), (33) reduces to

$$
\begin{aligned}
\dot{V}= & s\left(\tilde{a}_{1}\left(y_{1}-x_{1}\right)-\tilde{a}_{2}\left(y_{2}-x_{2}\right)\right)+s\left(-\tilde{b}_{2} y_{2}+\left(\tilde{b}_{1}-\tilde{a}_{1}\right) x_{1}+\tilde{b}_{1} y_{1}\right) \\
& +s\left(\tilde{c}_{2} z_{2}-\tilde{c}_{1} z_{1}-\frac{15 s}{|s|+0.01}\right)+\tilde{a}_{1}\left(-\left(y_{1}-x_{1}\right) s+x_{1} s\right)+\tilde{b}_{1}\left(-\left(x_{1}+y_{1}\right) s\right) \\
& +\tilde{c}_{1}\left(z_{1} s\right)+\tilde{a}_{2}\left(\left(y_{2}-x_{2}\right) s\right)+\tilde{b}_{2}\left(y_{2} s\right)+\tilde{c}_{2}\left(-z_{2} s\right) .
\end{aligned}
$$

Then (45) reduces to

$$
\dot{V}=-\frac{15 s^{2}}{|s|+0.01} \text {. }
$$

Since both $s^{2}>0$ and $|s|>0$, then, when $e \neq 0$, we have $\dot{V}<0$, hence $V$ is positive-definite while $\dot{V}$ is negative-definite. Recall the Lyapunov stability theory [18], then the response system (37) and the drive system (36) are synchronised globally and asymptotically. This completes the proof.

For simulations, the Adams-Bashforth-Moulton method is applied to solve the systems for the fractional order $p_{i}=0.95, i=1,2,3$, and the uncertain parameters are set to $a_{1}=$ $35, b_{1}=28, c_{1}=3, a_{2}=36, b_{2}=20$ and $c_{2}=3$. In the simulations, the initial values of the fractional-order drive and response systems (36)-(37), respectively, were arbitrarily set to $x_{1}(0)=6, y_{1}(0)=3, z_{1}(0)=7, x_{2}(0)=2, y_{2}(0)=7$ and $z_{2}(0)=4$; and the estimated parameters were set to $\tilde{a}_{1}(0)=1, \tilde{b}_{1}(0)=1, \tilde{c}_{1}(0)=1, \tilde{d}_{1}(0)=1, \tilde{a}_{2}(0)=1$ and $\tilde{b}_{2}(0)=1$. The modified adaptive sliding-mode synchronisation of the systems (36)-(37), obtained via the adaptive control laws (40) and (41), is shown in Figs. 3-4. Figures 3(a)-(c) display the steady-state plane trajectories of the drive (36) and response (37) systems, while Fig. 3(d) displays the synchronisation errors $e_{1}, e_{2}$ and $e_{3}$ as functions of time $t$. Figures 4(a)-(b) display the temporal response of the estimated parameter values $\tilde{a}_{1}, \tilde{b}_{1}, \tilde{c}_{1}, \tilde{a}_{2}$ and $\tilde{b}_{2}$ of the drive (36) and response (37) systems.

\section{Conclusion}

In this article, we have proposed a modified adaptive sliding-mode controller to realise chaos synchronisation between two different fractional-order chaotic systems with fully unknown parameters. The Lyapunov stability theory proved the asymptotic stability of the error system at the origin. The design of a suitable adaptive sliding-mode controller ensures target synchronisation. This work provides parameter update laws that estimate the true values of the unknown parameters. This paper also presents two numerical examples 

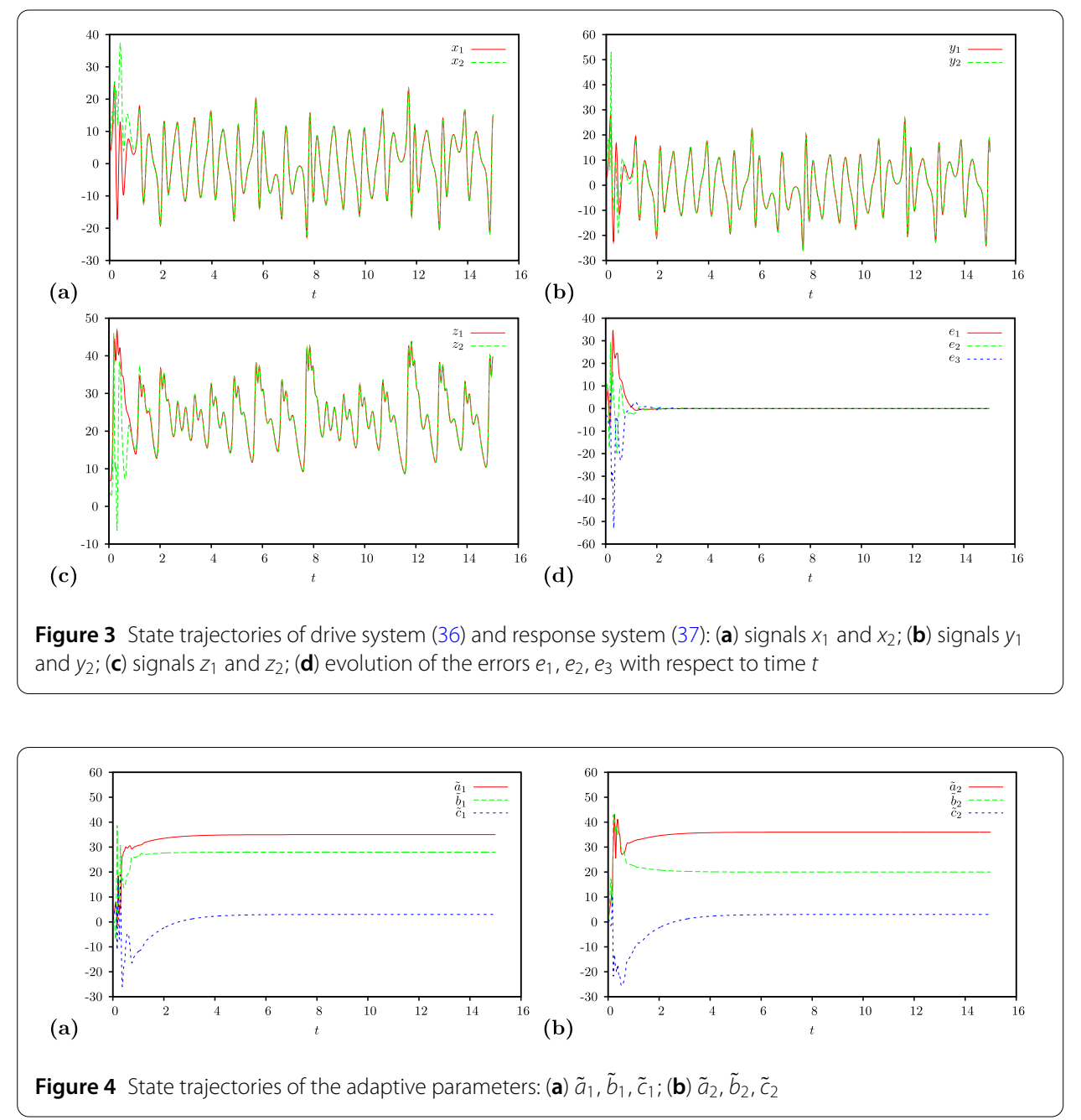

of different unknown fractional-order chaotic systems. Simulation results validate the efficiency and performance of the proposed modified adaptive sliding-mode synchronisation strategy. For future study, designing a modified adaptive sliding-mode controller to realise chaos synchronisation in drive-response dynamical networks is worth considering.

\section{Acknowledgements}

The author would like to thank the editor, associate editor and the anonymous reviewers for their many helpful comments and suggestions that have helped to improve the quality of this paper.

\section{Funding}

No funding is available.

Availability of data and materials

Not applicable.

Competing interests

The author declares that they have no competing interests.

\section{Authors' contributions}

The author read and approved the final manuscript.

\section{Publisher's Note}

Springer Nature remains neutral with regard to jurisdictional claims in published maps and institutional affiliations. 
Received: 19 November 2019 Accepted: 12 February 2020 Published online: 19 February 2020

\section{References}

1. Diethelm, K.: The Analysis of Fractional Differential Equations. Springer, Berlin (2010)

2. Yang, X.J.: Advanced Local Fractional Calculus and Its Applications. World Science, New York (2012)

3. Cattani, C., Srivastava, H.M., Yang, X.J.: Fractional Dynamics. De Gruyter, Berlin (2019)

4. Koeller, R.C.: Application of fractional calculus to the theory of viscoelasticity. J. Appl. Mech. 51, $299-307$ (1984)

5. Ichise, M., Nagayanagi, Y., Kojima, T.: An analog simulation of noninteger order transfer functions for analysis of electrode process. J. Electroanal. Chem. 33, 253-265 (1971)

6. Hao, Z., Shangbo, Z., Zhongshi, H.: Chaos synchronization of the fractional-order Chen system. Chaos Solitons Fractals 4, 2733-2740 (2009)

7. Al-Sawalha, M.M., Al-Sawalha, A.: Anti-synchronization of fractional order chaotic and hyperchaotic systems with fully unknown parameters using modified adaptive control. Open Phys. 14, 304-313 (2016)

8. Hajipour, A., Aminabadi, S.S.: Synchronization of chaotic Arneodo system of incommensurate fractional order with unknown parameters using adaptive method. Optik 127, 7704-7709 (2016)

9. Deepika, D., Sandeep, K., Shiv, N.: Uncertainty and disturbance estimator based robust synchronization for a class of uncertain fractional chaotic system via fractional order sliding mode control. Chaos Solitons Fractals 115, 196-203 (2018)

10. Tsung, C.L., Tun, Y.L., Valentina, E.B.: Adaptive fuzzy sliding mode control for synchronization of uncertain fractional order chaotic systems. Chaos Solitons Fractals 44, 791-801 (2011)

11. Ardashir, M.Z., Sehraneh, G., Okyay, K., Sohrab, K.M.: Robust predictive synchronization of uncertain fractional-order time-delayed chaotic systems. Soft Comput. 23, 6883-6898 (2019)

12. Ardashir, M.Z., Sehraneh, G.: Optimal synchronization of fractional-order chaotic systems subject to unknown fractional order, input nonlinearities and uncertain dynamic using type-2 fuzzy CMAC. Nonlinear Dyn.. 88, 2993-3002 (2017)

13. Ardashir, M.Z., Sehraneh, G.: Robust synchronization of uncertain fractional-order chaotic systems with time-varying delay. Nonlinear Dyn. 93, 1809-1821 (2018)

14. Sakthivel, R., Sakthivel, R., Nithya, V., Selvaraj, P., Kwon, O.M.: Fuzzy sliding mode control design of Markovian jump systems with time-varying delay. J. Franklin Inst. 355, 6353-6370 (2018)

15. Selvaraj, P., Kwon, O.M., Sakthivel, R.: Disturbance and uncertainty rejection performance for fractional-order complex dynamical networks. Neural Netw. 112, 73-84 (2019)

16. Agrawal, S.K., Das, S.: A modified adaptive control method for synchronization of some fractional chaotic systems with unknown parameters. Nonlinear Dyn. 73, 907-919 (2013)

17. Podlubny, I.: Fractional Differential Equations. Academic Press, New York (1999)

18. Liapunov, A.M.: Stability of Motion. Elsevier, New York (1966)

19. Petras, I.: Fractional-Order Nonlinear Systems Modeling, Analysis and Simulation. Springer, Berlin (2011)

20. Faieghi, M.R., Delavari, H.: Chaos in fractional-order Genesio-Tesi system and its synchronization. Commun. Nonlinear Sci. Numer. Simul. 17, 731-741 (2011)

21. Lü, J.G.: Chaotic dynamics of the fractional order Lü system and its synchronization. Phys. Lett. A 354, 305-311 (2006)

22. Lu, J.G., Chen, G.: A note on the fractional-order Chen system. Chaos Solitons Fractals 27, 685-688 (2006)

\section{Submit your manuscript to a SpringerOpen ${ }^{\circ}$ journal and benefit from:}

- Convenient online submission

- Rigorous peer review

Open access: articles freely available online

- High visibility within the field

- Retaining the copyright to your article

Submit your next manuscript at $\boldsymbol{s p r i n g e r o p e n . c o m ~}$ 